\title{
Detection of SV40 like viral DNA and viral antigens in malignant pleural mesothelioma
}

\author{
M. Ramael*,+, J. Nagels ${ }^{\#}$, H. Heylen ${ }^{\#}$, S. De Schepper", J. Paulussen ${ }^{\#}$, \\ M. De Maeyer $\#$, C. Van Haesendonck
}

Detection of SV40 like viral dna and viral antigens in malignant pleural mesothelioma. M. Ramael, J. Nagels, H. Heylen, S. De Schepper, J. Paulussen, M. De Maeyer, C. Van Haesendonck. C ERS Journal Ltd 1999.

ABSTRACT: This study investigated the presence of simian vacuolating virus 40 (SV40) deoxyribonucleic acid (DNA) in malignant pleural mesothelioma, non-neoplastic mesothelium and pleural carcinoma metastasis and correlated these data with immunohistochemistry for SV40 viral antigens.

The novel Primed In Situ (PRINS) method was applied to detect the presence of SV40 DNA in situ in tissue sections of malignant mesothelioma $(n=25)$, non-neoplastic mesothelium $(n=30)$ and pleural carcinoma metastasis $(n=30)$. Immunohistochemistry with an SV40-specific antibody was applied for detection of the SV40 viral antigen in the same material.

SV40 DNA and expression of one of the viral proteins (small t-antigen) was found in $\sim 60 \%$ of the investigated mesothelioma cases in contrast to non-neoplastic mesothelium and carcinoma metastasis that were negative for both SV40 DNA and SV40 viral antigens.

These results suggest that simian vacuolating virus 40 deoxyribonucleic acid may be biologically active as there was also immunoreactivity for simian vacuolating virus 40 viral antigen in those cases positive for simian vacuolating virus 40 deoxyribonucleic acid with the primed in situ reaction. Simian vacuolating virus 40 viral deoxyribonucleic acid and antigens may be potential markers for neoplastic mesothelium that may prove useful in the rather difficult histopathological differential diagnosis between malignant mesothelioma and reactive mesothelium or pleural carcinoma.

Eur Respir J 1999; 14: 1381-1386.

*Dept Pathology and "Dept Internal Medicine, General Hospital St. Elisabeth, Herentals, Belgium. 'Royal Antwerp Micrographical Society, Antwerp, Belgium.

Correspondence: M. Ramael

Dept Pathology

General Hospital St. Elisabeth

Nederrij 133

B-2200 Herentals

Belgium

Fax. 3237750433

Keywords: Immunohistochemistry mesothelioma

primed in situ method

primers

simian vacuolating virus 40

viral antigens

Received: January 131999

Accepted after revision July 21999
In 1960, Sweet and Hilleman reported the discovery of a new simian virus, simian vacuolating virus 40 (SV40) which grew in rhesus monkey cells. SV40 could infect human mesothelial cells in tissue culture and some of these cells became transformed [1]. The capacity of SV40 to induce tumours is mainly a function of both the SV40 large and small t-antigen [2]. These proteins promote transformation by binding and inactivating the products of several tumour suppressor genes. Except for a report demonstrating SV40 virus in one metastatic melanoma, SV40 was not considered to be oncogenic in humans [3]. Recently, some authors described the presence of SV40like deoxyribonucleic acid (DNA) in human tumours such as osteosarcomas, brain tumours, chorioid plexus tumours, and malignant mesothelioma [4]. The latter is a high grade malignant therapy resistant tumour originating from the serosal lining of the pleural, peritoneal or pericardial cavities. Asbestos was hitherto the only known agent to be associated with the development of mesothelioma [5]. Some laboratories, however, were unable to detect SV40 DNA by polymerase chain reaction (PCR) assays in human tissue including mesothelioma [6]. As conflicting results were reported, it was decided to investigate the presence of SV40 or SV40-like DNA in pleural mesothelioma, non-neoplastic mesothelium and pleural carcinoma metastasis with the Primed In Situ (PRINS) labelling technique permitting the visualization in situ the viral DNA in paraffin embedded formalin fixed tissue.

The PRINS method is based on a primer mediated DNA synthesis. It starts with the annealing of an oligonucleotide DNA primer adjacent to the DNA region of interest. The annealed oligonucleotide serves as a primer for the DNA polymerase T-aq polymerase. The T-aq polymerase incorporates the four nucleotides deoxyadenosine, deoxyguanosine, deoxyuridine and deoxycytidine triphosphate (dATP, dGTP, dUTP, and dCTP, respectively) of which the dUTP is labelled with digoxigenin (DIG) [7]. The label can be visualized immunohistochemically using alkaline phosphatase bound antibody. The application of the PRINS methodology has focussed primarily on cytogenetics [7]. Some applications were recently described dealing with the application of this method on paraffin embedded formalin fixed tissue [8].

This study investigated whether the PRINS technique with SV40 specific oligonucleotide DNA primers was able to detect SV40 DNA in paraffin embedded material. A second objective was to investigate whether the presence of SV40 also induced the presentation of viral antigens. Since the differential diagnosis between mesothelioma and 
pleural metastasis of carcinoma can be quite difficult on morphological basis alone, it was also investigated whether the presence of SV40-like DNA or viral proteins could be used as potential markers for neoplastic mesothelium.

\section{Materials and methods}

\section{Materials}

A total of 25 paraffin embedded mesothelioma tissue specimens comprising 12 epithelial mesotheliomas and 13 sarcomatoid mesotheliomas were included in the study together with 30 pleural tissue specimens or exudates with non-neoplastic mesothelium. Additionally, pleural tissues and pleural exudates were investigated from 30 patients with histologically proven carcinoma metastasis including 25 non-small cell lung cancer and five small cell lung cancers. Asbestos exposure was documented in all mesothelioma cases.

All tissues were routinely fixed in neutral formalin (4\%) and processed to a paraffin block with a classical histokinette (Bavimed, Bavaria, Germany.). Fixation times varied from 6-24 h. After sectioning and hematoxylin-eosin staining, periodic acid-Schiff (PAS), PAS after diastase pretreatment, alcian blue, and alcian blue after hyaluronidase treatment was performed. Immunohistochemical staining for carcinoembryonic antigen (CEA), vimentin, cytokeratin and epithelial membrane antigen (EMA) was performed, employing standard routine streptavidin-biotin immunohistochemical methods. Mesothelioma diagnosis was confirmed by the Belgian Mesothelioma Panel.

\section{Study design}

All the formalin-fixed paraffin-embedded tissues of patients with malignant pleural mesothelioma, carcinoma metastasis and nonneoplastic mesothelium were investigated with the PRINS method and immunohistochemistry for viral t-antigen. The PRINS reaction was carried out with three separate, primers: PYVrev, SV2for and SVrev detecting a DNA sequence coding for the viral T-antigen of SV40. These results were correlated with immunohistochemistry for viral antigens with the mouse monoclonal antibody Pab280 (dilution 1/20) (Oncogene, Cambridge, MA, USA). For each case at least 200 mesothelial cells or cells of pleural carcinoma metastasis were evaluated for SV40 small t-antigen and PRINS reactivity by two investigators.

\section{Preparation of paraffin sections}

Paraffin sections $5 \mathrm{~mm}$ thick were cut, floated on a protein-free water bath at $37^{\circ} \mathrm{C}$ and adhered on organosilane-coated glass slides (pretreated for $20 \mathrm{~min}$ with 3\% 3-aminopropyltriethoxysilan (Sigma, St Louis, MO, USA) in acetone). After drying at $37^{\circ} \mathrm{C}$ the slides were baked at $60^{\circ} \mathrm{C}$ for $2 \mathrm{~h}$ and stored dust-free.

\section{Primed in situ technique}

Prehybridization treatment. Each section was dewaxed in two rinses of xylene substitute Parasolve (Prosan, Ghent, Belgium), and rehydrated through a graded series of alcohol to water.
The tissue was then digested with pepsin $\left(4 \mathrm{mg} \cdot \mathrm{mL}^{-1}\right.$ in $0.2 \mathrm{~N}$ acetic acid; Sigma) solution for $45 \mathrm{~min}$ at $37^{\circ} \mathrm{C}$. After washing two times in distilled water, the sections were dehydrated in a graded series of alcohol to isopropylalcohol $100 \%$ and air dried.

Primed in situ technique. The sections were denatured in formamide solution at $94^{\circ} \mathrm{C}$ for $5 \mathrm{~min}$. The solution is prepared as follows: $70 \mathrm{~mL}$ deionized formamide, 10 $\mathrm{mL} 20 \times \mathrm{SSC}, 10 \mathrm{~mL}$ Sörensen buffer pH $7.0(0.2 \mathrm{M}$ $\left.\mathrm{NaH}_{2} \mathrm{PO}_{4} ; 0.2 \mathrm{M} \mathrm{Na}_{2} \mathrm{HPO}_{4}\right), 10 \mathrm{~mL}$ distilled water.

The sections were then washed in an ascending graded series of ice cold alcohol (isopropylalcohol 75\% 90\% and $100 \%$ ) at $-18^{\circ} \mathrm{C}$ for $3 \mathrm{~min}$ in each alcohol dilution.

The sections were air dried and immediately incubated dry at $90^{\circ} \mathrm{C}$ for $1 \mathrm{~min}$ using a hot plate (Medite, Burgdorf, Germany).

Preparation of primed in situ reaction mix. The following components were mixed in an eppendorf vial: $13 \mu \mathrm{L}$ distilled water, $3 \mu \mathrm{L}$ PRINS buffer $(10 \times$ concentrated; $50 \mathrm{mM} \mathrm{KCl}, 10 \mathrm{mM}$ TRIS-HCl, $1.5 \mathrm{mM} \mathrm{MgCl}, 0.01 \%$ bovine serum albumin ( $\mathrm{BSA})), 3 \mu \mathrm{L} 10 \times$ concentrated DIG-PRINS labelling mix $(500 \mu \mathrm{M}$ each of dATP, dCTP, and dGTP; $50 \mu \mathrm{M}$ DIG-dUTP; 50\% glycerol), 5 $\mu \mathrm{L}$ PRINS oligonucleotide primer (either positive control primer or SV40-specific oligonucleotide primer, 500 pmol), $1 \mu \mathrm{L} 450 \mu \mathrm{M}$ dTTP, $5 \mu \mathrm{L}$ Taq DNA polymerase (0.5 U. $\mu \mathrm{L})$.

All reagents were purchased from Boehringer-Mannheim (Mannheim, Germany) unless otherwise indicated The following primers sequences in brackets were used in separate reactions for SV40 detection: SVrev (GCATGACTCAAAAAACTTAGCAATTCTG), SV2 for (CTTTGGAGGCTTCTGGGATGCAACT) and PYVrev (GGAAAGTCTTTAGGGTCTTCTACC). The latter is detecting a region (4425-4402 base pairs (bp)) coding for Tantigen, which is common to the $\mathrm{BK}$ virus, $\mathrm{JC}$ virus and SV40 virus. The former two primers are SV40 specific. The SVrev primer is homologous to the 4399-4372 bp region and the SV2for primer is identical to the region 4920-4945 bp. Both are located in the DNA sequence coding for the t-antigen but outside the Rb-p107-Rb2/ p130 binding domain. [9]. A pan chromosome PRINS primer, specific for a universal motif of human alphoid sequences, allowing detection of all human chromosomes was used as a positive control for the PRINS reaction [7]. Cells of the cell line 293 were used as positive controls for the SV40 primers. Placental tissue was acting as a negative control. Primer was omitted in the negative control reactions.

Primer annealing and elongation. Each section at $94^{\circ} \mathrm{C}$ was covered with $30 \mu \mathrm{L}$ of PRINS mixture and covered with a cover slip. The target DNA was further denatured at $94^{\circ} \mathrm{C}$ for 3 min using a hot plate (Medite).

Primer annealing was carried out at $57^{\circ} \mathrm{C}$ (primers $\mathrm{SVrev}$ and SV2for) or $52^{\circ} \mathrm{C}$ (primer PYVrev) for $10 \mathrm{~min}$ followed by an elongation step at $72^{\circ} \mathrm{C}$ for $30 \mathrm{~min}$.

The hybridization/elongation reaction was stopped by washing the slides for 3-5 $\mathrm{min}$ in prewarmed washing buffer $(50 \mathrm{mM} \mathrm{NaCl} 50 \mathrm{mM}$ EDTA $\mathrm{pH} 8.0)$ at $55^{\circ} \mathrm{C}$.

Visualization of the PRINS reaction DNA with the alcaline phosphatase technique. The sections were washed 
three times for 3 min with phosphate buffered saline (PBS) $\mathrm{pH}$ 7.4. The sections were consecutively incubated for $30 \mathrm{~min}$ with: monoclonal anti-DIG-antibody labeled with fluorescein immunoglobulin (Ig)G Fab fragments (Boehringer-Mannheim) diluted 1:1,000 in PBS at $37^{\circ} \mathrm{C}$; sheep polyclonal anti-fluorescein-antibody labeled with alkaline phosphatase (Boehringer-Mannheim) diluted 1:1500 in PBS at room temperature; Alkaline phosphatase was visualized after incubation with fast red solution (5 mg fast red in $15 \mathrm{~mL}$ napthol buffer) (Biogenex, San Ramon, CA, USA) for 20 min according to the instructions of the manufacturer.

\section{Immunohistochemistry for SV40 T-antigen}

Paraffin sections (5-mm thick) were cut and dewaxed in parasolve, xylene substitute (Prosan) followed by rehydratation in a decreasing graded ethanol series, water and PBS, $\mathrm{pH}$ 7.4. Slides were incubated with nonimmune polyclonal rabbit serum (diluted 1:20 in PBS) (DAKO, Glostrup Denmark) for 20 min to block the nonspecific Fcreceptor activity in the tissue.

The sections were treated with: mouse monoclonal antiSV40 T-antigen antibody Pab 280 (Oncogene, USA) diluted 1:20 in PBS for $20 \mathrm{~min}$. The mouse monoclonal antibody (isotype $\operatorname{IgG}_{1}$ ) is directed against an epitope located on the $21 \mathrm{kDa}$ small antigen of SV40. This antibody that works on formalin fixed paraffin embedded tissue is specific for the small antigen of SV40 and does not cross-react with the large T-antigen [10].

\section{Visualization of bound antibody with the alcaline phos-} phatase technique

The sections were incubated for 20 min with DAKO Envision Plus dextran polymer complex bound to alkaline phosphatase followed by fast red as chromogen according to the guidelines of the manufacturer (DAKO).

Sections were washed in demineralized water, air dried and mounted with Imsol (Klinipath, Duiven, the Netherlands). The specificity of the reactions was controlled by omitting the first antibody and substituting the anti-SV40 antibody for an unrelated antibody of the same isotype at the same concentration. Formalin-fixed and paraffin-embedded 293 cells were used as a positive control. Formalinfixed and paraffin-embedded placental tissue was used as negative control tissue.

\section{Results}

There was bright finely granular nuclear staining in neoplastic mesothelial cells with the PRINS method. Nuclear staining was found in 14 of the 25 investigated mesothelioma tissue specimens using the PYVrev primer, indicating the presence of SV40 or SV40-like DNA, as this primer also recognizes a sequence identical to the SV40, JC and BK virus. A separate PRINS reaction with the SV2for primer or SVrev primer, that is specific for the SV40 virus, revealed nuclear signals in all those cases that tested positive with the PYVrev primer (fig. 1). The percentage of positive cells varied considerably between different tumours (table 1). The percentage of positive neoplastic cells ranged from $2 \%$ in an epithelial meso-

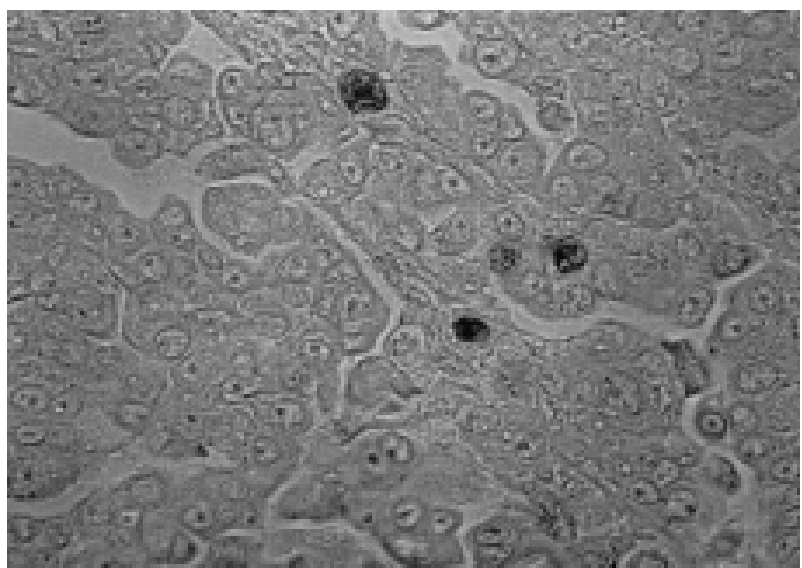

Fig. 1. - Some nuclei of neoplastic mesothelial cells display nuclear staining for simian vacuolating virus 40 (SV40) deoxyribonucleic acid (DNA) with the primed in situ (PRINS) method in an epithelial mesothelioma (SV2for primer). The majority of nuclei remains unstained. (Internal scale bar $=10 \mu \mathrm{m}$.)

thelioma to nearly $100 \%$ in a mesenchymal mesothelioma. Stromal elements such as blood vessels, adipocytes and inflammatory cells were found not to be positive. In all the cases that tested positive for SV40 or SV40-like DNA, cytoplasmic and nuclear immunoreactivity was found for the small t-antigen with the monoclonal antibody pAb280 (fig. 2). The amount of immunoreactive cells was comparable to those with the nuclear signal for SV40-like DNA in a given sample (table 1). Positive PRINS control reaction with the pan chromosome primer specific for all human chromosomes displayed in neoplastic mesothelial cells of every mesothelioma case multiple nuclear signals indicating the detection of chromosomal DNA (fig. 3). The 293 cells acting as positive controls were clearly positive with the PRINS reaction detecting the SV40 DNA and displayed cytoplasmic and nuclear immunoreactivity for small t-antigen with the monoclonal antibody Pab280.

Table 1. - Results of the primed in situ (PRINS) and immunohistochemical investigation for simian vacuolating virus 40 (SV40) deoxyribonucleic acid (DNA) and SV40 viral protein expressed as a percentage of positive neoplastic mesothelial cells

\begin{tabular}{lcc}
\hline $\begin{array}{l}\text { Mesothelioma } \\
\text { Subtype }\end{array}$ & $\begin{array}{c}\text { SV40 DNA } \\
\text { positivity }\end{array}$ & $\begin{array}{c}\text { SV40 viral protein } \\
\text { immunoreactivity }\end{array}$ \\
\hline Epithelial & & \\
Case 1 & 2 & 2 \\
Case 2 & 30 & 28 \\
Case 3 & 45 & 43 \\
Case 4 & 53 & 50 \\
Case 5 & 72 & 60 \\
Case 6 & 85 & 79 \\
Case 7 & 93 & 80 \\
Sarcomatoid & & \\
Case 8 & 2 & 2 \\
Case 9 & 23 & 20 \\
Case 10 & 43 & 37 \\
Case 11 & 60 & 50 \\
Case 12 & 74 & 70 \\
Case 13 & 92 & 89 \\
Case 14 & 95 & 98 \\
\hline
\end{tabular}




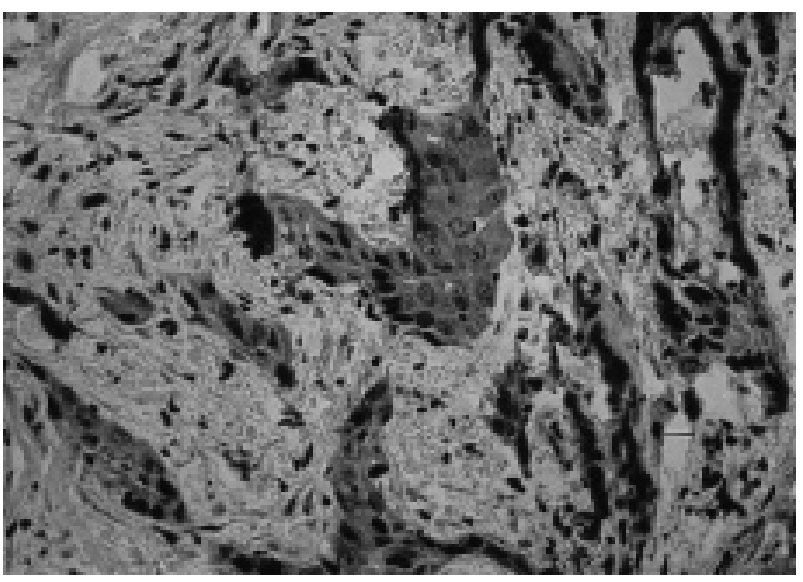

Fig. 2. - Nuclear and cytoplasmic immunoreactivity for small simian vacuolating virus 40 t-antigen in an epithelial mesothelioma with the monoclonal antibody pAb 280 in the majority of neoplastic cells. (Internal scale bar $=15 \mu \mathrm{m}$.)

No nuclear signal for SV40 or SV40-like DNA was found in the cases with carcinoma mestastasis or in the pleural effusions with non-neoplastic mesothelium. These cases were also found not to be immunoreactive for SV40 small t-antigen. Negative controls remained unstained.

\section{Discussion}

In this study, SV40 or SV40-like DNA was found in $\sim 60 \%$ of the investigated cases. In contrast, non-neoplastic mesothelium and pleural metastasis were not positive with this method. The malignant mesotheliomas presumed to harbour SV40 DNA were also found to be immunoreactive with the monoclonal antibody detecting the viral small tantigen of SV40. This is an indication that the viral SV40 DNA is being translated to viral oncoprotein in malignant mesothelioma. Both nuclear and cytoplasmic immunoreactivity were found with the monoclonal antibody $\mathrm{Pab}$ 280. This is not surprising, as the monoclonal antibody Pab 280 , specifically detecting the small t-antigen, is found to

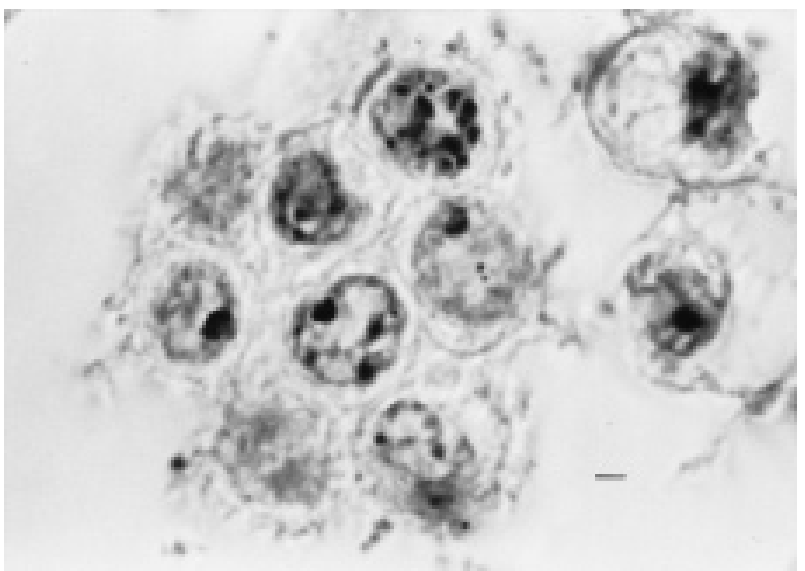

Fig. 3. - Positive primed in situ (PRINS) control reaction with the pan chromosome primer specific for all human chromosomes showing multiple nuclear signals indicating the detection of chromosomal deoxyribonucleic acid (DNA) in neoplastic mesothelial cells of an epithelial mesothelioma. (Internal scale bar $=5 \mu \mathrm{m}$.) react strongly with both the nuclear and cytoplasmic form of the small t-antigen that appears to be associated with the cytoskeleton thereby explaining the observed nuclear and cytoplasmic immunoreactivity in the present series [10].

However, cytoplasmic immunoreactivity has also been described for other classical nuclear antigens such as cmyc, the cellular homologue of the v-myc antigen. Fixation times that are longer than $60-120 \mathrm{~min}$ seem to influence the cellular localization of nuclear proteins such as c-mye and result in cytoplasmic immunoreactivity [11]. This phenomenon has also been described in paraffin embedded formalin fixed tissue of human pleural mesothelioma for c-fos and c-mye nuclear antigens [12].

Small t-antigen might play a key role in the development of mesothelioma as small t-antigen mutants are not able to induce mesothelioma when injected intrapleurally in hamsters in contrast to wild type SV40 [13]. Small tantigen enhances the transforming capacities of SV40 by increasing the production of t-antigen: by contributing to the complete inactivation of cellular p53, stimulating mitosis in quiescent cells [2]. Most mesotheliomas are found to be immunoreactive for the wild type p53 protein and retinoblastoma protein $[14,15]$. As mutation of the $p 53$ gene in malignant mesothelioma is very rare [16], in vitro experiments suggest that this protein is complexed with the SV40 viral t-antigen [17].

Recent investigations strongly corroborate the hypothesis that the expression of SV40 viral antigens and their interaction with the suppressor gene products of the $p 53$ gene and the retinoblastoma gene are one of the major steps to immortalize and transform mesothelial cells [18]. Immortalized cells are usually not able to induce tumours but may become oncogenic if they are transfected with an oncogene, or if they are treated with a carcinogen such as asbestos. A synergistic effect between asbestos and SV40 was suggested by the fact that asbestos facilitates transformation of cells in culture by SV40 [19]. In tumours developing in SV40 transgenic mice and in human cells in culture, transient expression of t-antigen seems to be sufficient to induce transformation. Interaction of viral antigens with $p 53$ would lead to genomic instability which may be sufficient to promote random chromosomal aberrations which maintain the neoplastic phenotype even at very low levels of t-antigen expression $[4,20]$. This might explain why in this study, a wide range of immunoreactive neoplastic cells for viral t-antigen were found.

The presence of SV40 DNA and the viral SV40 protein may serve as a potential marker for neoplastic mesothelium as pleural carcinoma mestastasis and nonneoplastic mesothelium were not positive for SV40 DNA or viral proteins in this study. These findings are in complete agreement with the findings of CARBONE et al. [4, 20] who did not detect SV40 or SV40-like DNA in both small cell and non-small cell lung cancer using a PCR assay.

Recently, Galateau-Salle et al. [21] described the presence of SV40 or SV40-like DNA sequences in mesothelioma, bronchial carcinoma and nonneoplastic pulmonary disease using a PCR based assay. PCR based assays for detection of SV40 virus must be interpreted with care as some PCR primers seem to amplify what appears to be host sequences. Unfortunately these PCR results were not confirmed by positive immunohistochemistry. It is unclear in this study whether the presence of the SV40 or SV40-like DNA sequences by the PCR 
assay is just fortuitous as both neoplastic tissue and nonneoplastic tissue was positive for SV40 or SV40 like DNA. The major advantage of the PRINS method is that it combines morphology with molecular information. Not only does it detect, but also visualizes the DNA thereby permitting its localization in a specific cell. In contrast, classical PCR only gives us an overall impression about the SV40 DNA content of a given tissue and does not permit the localization of the viral DNA. If false positive results occur, it is more likely that these will be more readily apparent with a morphological method such as PRINS than with classical PCR.

In addition to tumours, SV40 DNA has been detected in human pituitary gland, peripheral mononuclear blood cells and seminal fluids from healthy subjects [22] thereby explaining the results in the study of GaLATEAU-SALLE $e t$ al. [21]. The transmission of SV40 virus is not very clear at present. Some studies suggest that SV40 might be a contaminant of polio vaccines administerd in the years 1950-1960. The exact nature of the SV40 or SV40-like DNA revealed by PCR assays is still an enigma. Some primer sets result in a higher percentage of positive results than others [4]. The primers specific for the Rb-pocket give the highest positive results. Preliminary data suggest that primers considered specific for SV40 would, under certain conditions, amplify what appeared to be host DNA sequences, indicating that PCR based assays must be interpreted with caution and correlated with expression of viral T-proteins [18]. SV40 might be an endemic virus in the human population and be occasionally present in human tissues in low copy numbers.

Such endemic viruses may accumulate various mutations and deletions. Mutations and deletions of the SV40 genome may also occur in human tumours but are very unlikely to occur in the $\mathrm{Rb}$ pocket binding domain of $\mathrm{t}$ antigen because of the relevance of this region in cell transformation $[23,24]$. It is also possible that the sequence detected by these sets of primers belong to a recombinant between SV40 and another unknown virus. This hypothesis is corroborated by the fact that human specimens which contain SV40 are also coinfected with the BK virus. Furthermore, the adenovirus 3 and 7 vaccines used between 1961 and 1965 contained adenovirusSV40 hybrids with segments of the SV40 genome which included the SV40 t-antigen enclosed in their recombinant genome [4].

These findings indicate that some SV40 sequences including oncogenic sequences coding for t-antigen can be transferred and incorporated into the genome of another virus. The latter virus acting as a carrier is transformed into an oncogenic virus. In this way "unharmful" viruses such as adenoviruses serving as a carrier may spread oncogenic DNA sequences in the human population.

It is concluded that the primed in situ method is able to detect simian vacuolating virus 40 or simian vacuolating virus 40-like deoxyribonucleic acid in the majority of mesothelioma in contrast to non-neoplastic mesothelium and pleural carcinoma mestastasis that were simian vacuolating virus 40 deoxyribonucleic acid- and simian vacuolating virus 40 protein-negative. The simian vacuolating virus 40 deoxyribonucleic acid seems to be translationally active since all the positive cases were also immunoreactive for viral proteins. These preliminary results suggest that viral simian vacuolating virus 40 antigens might serve as a potential marker for neoplastic mesothelium, but these findings have to be confirmed with a larger series of mesothelioma cases.

Acknowledgements. The authors would like to thank K. Dugardeyn and K. Waelput (Omnilabo, Belgium) for technical assistance with the photographical work.

\section{References}

1. Ke Y, Reddel R, Gerwin B, Reddel H, Somers A, McMenamin M. Establishment of human in vitro mesothelial cell model system for investigating mechanisms of asbestos induced mesothelioma. Am J Pathol 1989; 134: 979-991.

2. Fanning E, Knipers R. Structure and function of SV40 large tumour antigen. Annu Rev Biochem 1992; 61: 5585 .

3. Soriano F, Shelburne E, Gokcen M. Simian virus 40 in a human cancer. Nature 1974; 249: 421-424.

4. Carbone M, Rizzo P, Pass HI. Simian virus 40, poliovaccines and human cancer: a review of recent developments. Oncogene 1997; 15: 1877-1888.

5. Roggli VL. Pathology of asbestos and associated diseases. Roggli VL, Grenberg SD, Pratt PC, eds. Little Brown Co. Press, Boston, 1992.

6. Strickler H, Goedert JJ, Fleming M, Travis W, Williams A, Rabkin C. Simian virus 40 and pleural mesothelioma in humans. Cancer epidemiology, Biomarkers and prevention. 1996; 15: 473-475.

7. Koch J, Kolvraa S, Peetersen P, Gregersen N, Boland L. Oligonucleotide priming methods for the chromosome specific labeling of alpha satellite DNA in situ. Chromosomea (Berlin) 1989; 98: 259-265.

8. Ramael M, Van Steelandt H, Stuyven G, Van Steenkiste M, Degroote J. Application of the Primed In situ Labeling (PRINS) method for detection of numerical chromosomal aberrations in paraffin embedded formalin fixed tissue of molar and non-molar pregnancies. Biochemica 1997; 2: $18-20$.

9. Bergsagel D, Finegold M, Butel J, Kujsky W, Garcea R. DNA sequences similar to those of SV40 in ependymomas and chorioid plexus tumours of childhood. $N$ Engl J Med 1992; 326: 988-993.

10. Montano X, Lane DR. Monoclonal antibody analysis of simian virus 40 small T-antigen in infected and transformed cells. $J$ Virol 1989; 63: 3128-3134.

11. Loke S, Neckers L, Schwab G, Jaffe S. c-Myc protein in normal tissue: effects of fixation on its apparent subcellular distribution. Am J Pathol 1988; 131: 29-37.

12. Ramael M, Van den Bossche J, Buysse C, Deblier L, Segers K, Van Marck E. Immunoreactivity for c-fos and c-myc protein with the monoclonal antibodies 14E10 and 6E10 in malignant mesothelioma and non-neoplastic mesothelium. Histol and Histopathol 1995; 10: 639-640.

13. Cicala C, Pompetti F, Carbone M. SV40 induces mesotheliomas in hamsters. Am J Pathol 1993; 142: 15241533.

14. Ramael M, Eerdekens C, Lemmens G, Deblier I, Jacobs W, Van Marck E. Immunoreactivity for $\mathrm{p} 53$ protein in neoplastic and non-neoplastic mesothelial tissues. $J$ Pathol 1992; 168: 371-375. 
15. Ramael M, Segers K, Van Marck E. Differential immunohistochemical staining for retinoblastoma protein in malignant mesothelioma with the antibodies $\mathrm{C} 15$ and $1 \mathrm{~F} 8$. Pathol Res Pract 1994; 190: 138-141.

16. Segers K, Backhovens H, Kumar S, et al. Immunoreactivity for $\mathrm{p} 53$ and $\mathrm{mdm} 2$ and the detection of $\mathrm{p} 53$ mutations in human malianant mesothelioma. Virchows' Arch Pathol 1996; 427: 431-436.

17. De Mluca A, Baldi A, Esposito V, Howard C, Bagella L, Rizzo P. The retinoblastoma gene family $\mathrm{pRb} / \mathrm{p} 105$, $\mathrm{p} 1087, \mathrm{pRb} 2 / \mathrm{p} 130$ and simian virus $40 \mathrm{~T}$ antigen in human mesotheliomas. Nat Med 1997; 18: 913-916.

18. Centers for disease Control. Simian Virus 40(SV40), a possible human polyomavirus. Em Infect Dis 1997; 3: 245-247.

19. Appel J, Fasy T, Kohtz D. Asbestos fibers mediate transformation of monkey cells by exogenous DNA. Proc Natl Acad Sci USA 1988; 85: 7670-7674.
20. Carbone M, Rizzo P, Pass H. Simian virus 40-like DNA sequences in human pleural mesothelioma. Oncogene 1994; 9: 1781-1790.

21. Galateau-Salle F, Bidet Ph, Iwatsubo Y, et al. SV40-like DNA sequences in pleural mesothelioma, bronchopulmonary carcinoma and non-malignant pulmonary disease. J Pathol 1998; 184: 252-257.

22. Martini F, Iaccheri I, Lazzerin L, Carinci R, Corallini A, Gerosa M. SV40 early region and large T antigen in human brain tumours, peripheral blood cells and sperm fluids from healthy individuals. Cancer Res 1996; 56 : 4820-4825.

23. Morwood K, Price TNC, Mayne LV. Mutation of p53 is not a prerequisite for immortalization of human fibroblasts by SV40 T antigen. Exp Cell Res 1996; 223: 308-313.

24. Carbone M, Rizzo P, Procopio A, et al. SV40 like sequences in human bone tumours. Oncogene 1996; 13: $527-535$. 